\title{
Termination of the normal conus medullaris in children: a whole-spine magnetic resonance imaging study
}

\author{
Henry Kesler, M.D., ${ }^{1}$ Mark S. Dias, M.D., ${ }^{1}$ And Paul Kalapos, M.D. ${ }^{1,2}$ \\ Departments of ${ }^{\prime}$ Neurosurgery and ${ }^{2}$ Radiology, Pennsylvania State University College of Medicine, \\ Penn State Milton S. Hershey Medical Center, Hershey, Pennsylvania
}

\begin{abstract}
Object. The mean level of the conus medullaris (CM) has been estimated to lie opposite the L1/2 disc space in several previous studies using ultrasound, CT myelography, and magnetic resonance (MR) imaging, but these studies have been limited in examining only the lumbar spine and including patients being evaluated for back pain and sciatica (creating a selection bias). Moreover, significant variability was found in the termination of the CM, with a small subset of subjects having a CM as low as the mid-body of L4. The authors sought to determine the normal level of the CM and its variability.

Methods. Children with brain or spinal cord tumors who underwent whole-spine surveillance MR imaging were identified retrospectively. The level of the CM was identified in each subject by counting down from $\mathrm{C} 1$. Vertebral anomalies, such as lumbarized S1, sacralized L5, or fewer rib-bearing segments, and the presence of fatty filum were noted.

Results. Findings regarding the level of termination of the CM were tightly grouped; the average was at the lower third of L1 and the mode of the distribution was at the L1/2 disc space, with very little variation. No CM ended below the mid-body of L2. The level of the CM was not significantly different among individuals with lumbarized or sacralized vertebrae or 11 rib-bearing segments.

Conclusions. The CM terminates most commonly at the L1-2 disc space and in the absence of tethering, the CM virtually never ends below the mid-body of L2. A CM that appears more caudal on neuroimages should be considered tethered. (DOI: $10.3171 /$ FOC-07/08/E7)
\end{abstract}

\section{KEY WordS • conus medullaris - magnetic resonance imaging • pediatric neurosurgery • spinal cord • tethered cord}

$\mathrm{T}$ HE ASCENT OF THE CM with respect to the adjacent vertebral column during fetal and postnatal development and its ultimate level of termination postnatally have been the subject of several studies using both cadavers and various imaging modalities. Hawass and colleagues, ${ }^{5}$ in a study of 340 aborted fetuses using lumbar myelography, chronicled a progressive ascent of the CM between 12 and 33 weeks' gestation, with all fetuses having a CM opposite or cranial to the L3 vertebral level by 33 weeks' gestation. Barson, ${ }^{1}$ in an anatomical study of aborted fetuses, also documented a progressive rise in the level of the CM between the 12th fetal week and postnatal development, with the $\mathrm{CM}$ coming to lie opposite or cranial to the L2/3 disc space by 40 weeks' gestation and opposite or cranial to the L1/2 disc space by 2 months after birth. Beek and associates, ${ }^{2}$ using ultrasound in 99 infants, some of whom were born prematurely, determined the CM to lie opposite the L2/3 disc space at 27-29 weeks' of gestation and opposite the L1/2 disc space by 40 weeks' gestation. James and Lassman, ${ }^{7}$ in a study of 25 cadavers ranging in age from birth to 8 years, found that the $\mathrm{CM}$ reaches an average level opposite the mid-body of

\footnotetext{
Abbreviations used in this paper: $\mathrm{CI}=$ confidence interval; $\mathrm{CM}=$ conus medullaris; $\mathrm{MR}=$ magnetic resonance PSHMC = Penn State Hershey Medical Center.
}

L2 by 5 months after birth.Wolfe and colleagues, ${ }^{14}$ in a study of 55 term infants examined by ultrasound, found that in $94 \%$ the CM ended opposite or cranial to the L2 vertebra. Jit and Charnalia8 also established the normal level of the CM opposite or cranial to the L2 vertebra in newborn infants. It is evident from these studies that the $\mathrm{CM}$ reaches its final level between birth and 5 months of age and does not ascend further beyond this time.

Investigators have examined the level of the CM in infants, children, and adults using ultrasound or MR in a number of studies (Table 1).,3,6,9-14 The average level of termination of the $\mathrm{CM}$ in these studies was between the mid-body of $\mathrm{L} 1$ and the $\mathrm{L} 1 / 2$ disc space. The range varied between the T10/11 disc space and the mid-body of L4, however, with a CM opposite or cranial to the L2/3 disc space being accepted as "normal" in all of the studies.

These previous studies all have one or more significant limitations. First, the authors used only lumbar spine images and "counted up" from the sacrum; neither the number of cervical vertebrae nor the number of rib-bearing vertebrae was assessed. Second, patients with transitional, lumbarized, or sacralized vertebrae were specifically excluded from these studies. Third and most important, the authors included many patients who underwent imaging studies for evaluation of back pain or "sciatica," creating an obvious selection bias. 
H. Kesler, M. S. Dias, and P. Kalapos

TABLE 1

Level of termination of the CM reported in previous imaging studies*

\begin{tabular}{lcccc}
\hline \hline \multicolumn{1}{c}{ Author(s) \& Year } & Population & Imaging Modality & Average & Range \\
\hline Wilson et al., 1989 & 184 infants & MRI & L1/2 & T12-L2/3 \\
Hill \& Gibson, 1994 & 103 infants & ultrasound & L2U & T12/L1-L3M \\
Wolfe et al., 1992 & 114 infants & ultrasound & L1/2 & T12-L4 \\
Sahin et al., 1997 & 64 infants & ultrasound & L1/2 & T12/L1-L2/3 \\
Tame \& Burstal, 2003 & 45 children & MRI & L1 & T11/12-L2/3 \\
DiPietro, 1993 & 161 children & ultrasound & L1 & T10/11-L3U \\
Demiryürek et al., 2002 & 639 adults & MRI & L1M & T11/12-L3U \\
Lee et al., 2004 & 210 adults & MRI & L1L & T12/L1-L2U \\
Saifuddin et al., 1998 & 504 adults & MRI & L1L & T12M-L3U \\
\hline
\end{tabular}

* The vertebral terminology used in this paper is explained in Materials and Methods. Abbreviations: $\mathrm{U}=$ upper; $\mathrm{M}=$ middle; $\mathrm{L}=$ lower.

This present study was undertaken in a population of children with brain tumors who were undergoing wholespine surveillance imaging to exclude leptomeningeal seeding with the intention of 1) determining both the normal level and variability of termination of the $\mathrm{CM}$; 2) assessing the level of termination of the CM in children with transitional vertebrae and those with abnormal numbers of rib-bearing segments; and 3) establishing changes, if any, in the level of termination of the $\mathrm{CM}$ in children over time on serial imaging studies.

\section{Clinical Material and Methods}

All children with brain and/or spinal cord tumors treated between 1991 and 2005 at the PSHMC were identified from a hematology-oncology database. All children who had undergone whole-spine MR imaging at PSHMC to assess for leptomeningeal seeding were selected for review. Patients with leptomeningeal seeding were included as long as the seeding did not compress the spinal cord or

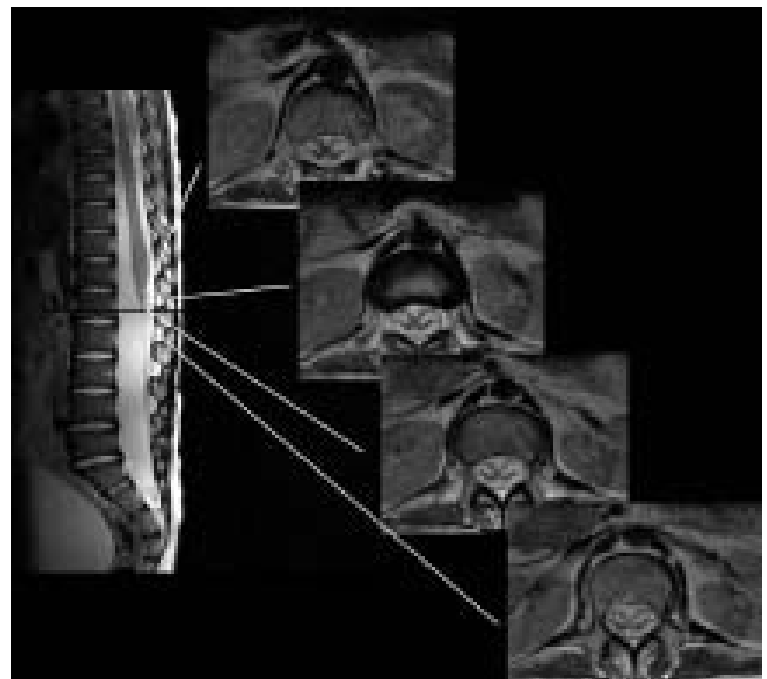

FIG. 1. Method of determining the termination of the CM on sagittal and axial MR images. A: Mid sagittal T2-weighted MR image showing the tapering CM ending at the lower section of the body of L1. Corresponding axial images depict spinal cord levels cranial to (upper two images), at (third image), and caudal to (lower image) the tip of the CM. interfere with the proper identification of the CM. Patients with tumors or leptomeningeal seeding that involved the conus medullaris or cauda equina were excluded. No patient had any identifiable cutaneous manifestations of spinal dysraphism.

All MR images were reviewed by one of the senior authors (M.D. or P.K.) in conjunction with the third author (H.K.). The termination of the CM was defined as the level of the lowest axial image in which spinal cord tissue was identifiable (Fig. 1). Axial and sagittal MR images were correlated in each case to confirm the level of termination of the CM. The termination of the $\mathrm{CM}$ was identified relative to the adjacent vertebrae, with each vertebral body divided into upper, middle, and lower thirds and the intervertebral disc space serving as a fourth level. For the purpose of this paper, we represent these positions by showing the vertical body followed by $\mathrm{U}$ for upper, $\mathrm{M}$ for medium, and $\mathrm{L}$ for lower and designating the vertebral disc space with a virgule (for example, T11/12). For each imaging study, we identified the number of cervical and rib-bearing segments by counting down from the atlas, and the number of lumbar vertebrae were counted from the lumbosacral junction. Transitioning from cervicothoracic to thoracolumbar images was done by identifying anatomical landmarks (vascular markings, unique vertebral markings, or diaphragmatic attachments) common to both studies, rather than by using gelatin capsules (which would have yielded incorrect registrations in two children). The last rib-bearing vertebra was established by identifying the rib on coronal and/or axial images (Fig. 2). The first sacral vertebral segment was identified by the presence of the sacroiliac joint on coronal images (Fig. 2C). The presence of fewer than 12 ribs, a sacralized fifth lumbar vertebra, or a lumbarized first sacral vertebra was noted. The presence of fat within, or thickening of, the terminal filum was also recorded.

For calculation purposes, each assigned section of the vertebral column was given a numerical score ranging from 1 (representing the upper border of T12 [T12U]) to 11 (representing the lower border of L2 [L2L]) as represented in Fig. 3. The mean, median, mode, and range, as well as standard deviation and $95 \% \mathrm{CI}$, were then calculated from these numerical values.

The first imaging studies used for each patient were all performed before, or very shortly after the initiation of therapy; there should have been no effects of treatment on 


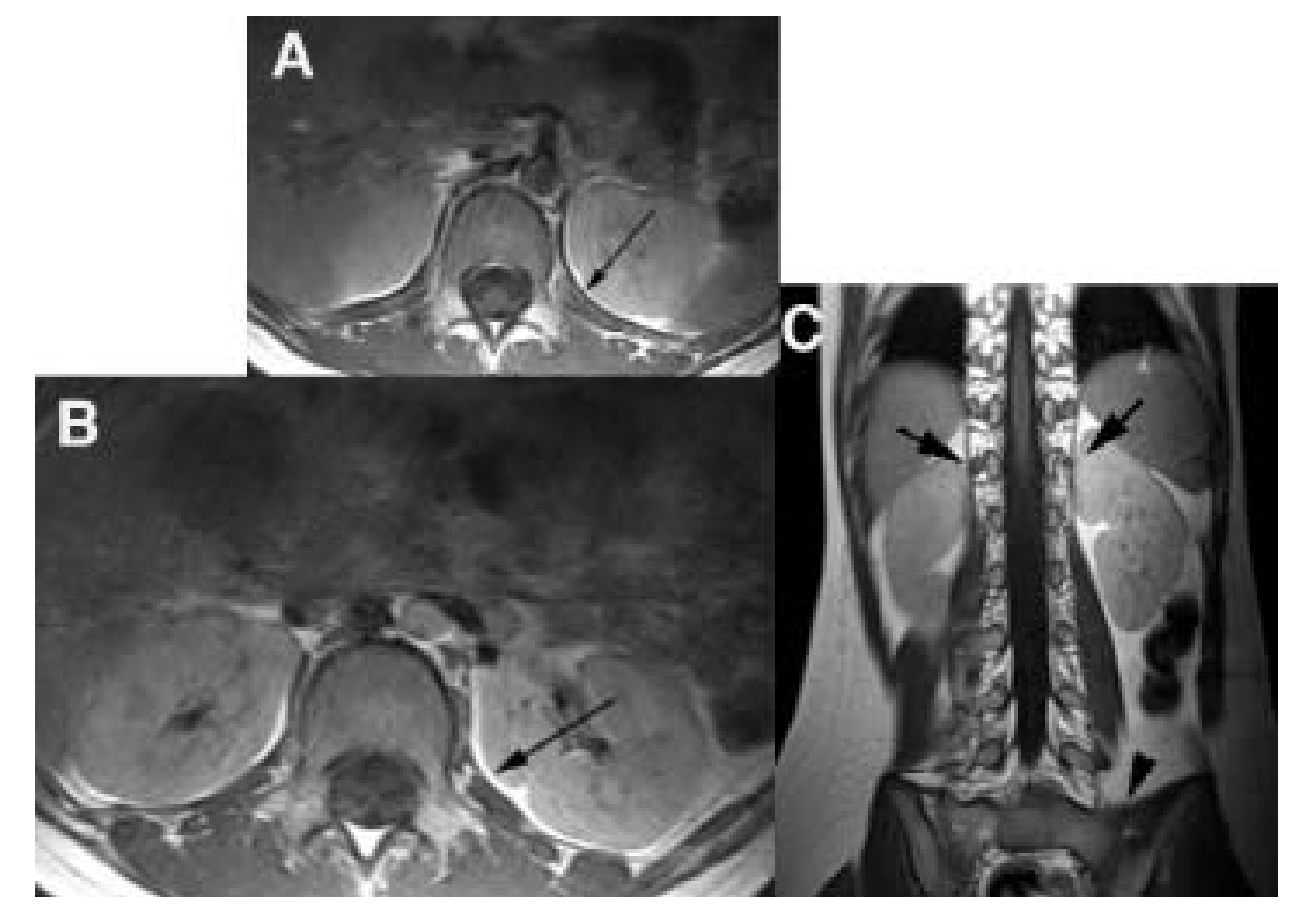

FIG. 2. Identification of rib and sacroiliac joints. A: Axial T1-weighted MR image showing T12 with the attached rib (arrow). B: Axial T1-weighted MR image obtained at the L2 level showing no rib. C: Coronal T1-weighted MR image showing the articulating rib heads (arrows) and the sacroiliac joint between S1 and the ilium, particularly on the left (arrowhead).

vertebral body height or growth when these studies were performed. All images from subsequent studies were similarly evaluated and compared with the initial MR images to identify any changes in the level of the CM over time.

\section{Results}

One hundred children who underwent whole-spine imaging were identified during the study period. Their average age was 7.5 years (range $0.4-18$ years). The group included 51 boys and 49 girls. Their tumors represented a variety of histological diagnoses, the most common being medulloblastoma and astrocytoma (including benign and anaplastic astrocytomas).

The level of the CM in these 100 children is graphically represented in Fig. 4. All the CMs terminated between the lower third of T12 (T12L) and the middle of L2 (L2M). The mean corresponded to the lower third of L1 (L1L) and the mode corresponded to the L1/2 disc space. The standard deviation was 1.59 sections, yielding a $95 \%$ CI between the lower third of T12 (T12L) and the middle section of the L2 vertebra. In no child was the CM shown to be below the middle third of L2 (L2M) on the initial MR images.

Two serial MR images were evaluated in 46 children. In 37 of these children, the level of the CM did not change between the two studies. In three children the CM was higher by one section (one third of a vertebra) and in six children the CM was lower by one section. In a single child the CM was observed to have descend from the middle third of L2 to the lower third of L2 between studies. The amount of change was insignificant and likely to have been within the error of measurement.
Transitional vertebrae were identified in six children, all having lumbarized S1 vertebrae. Counting the last ribbearing segment as $\mathrm{T} 12$, the termination of the $\mathrm{CM}$ in these six patients was not significantly different from that in the group as a whole- the CM ended opposite L1L in one child, L2U in another child, and the L1/2 disc space in the remaining four children. Two children had 11 rib-

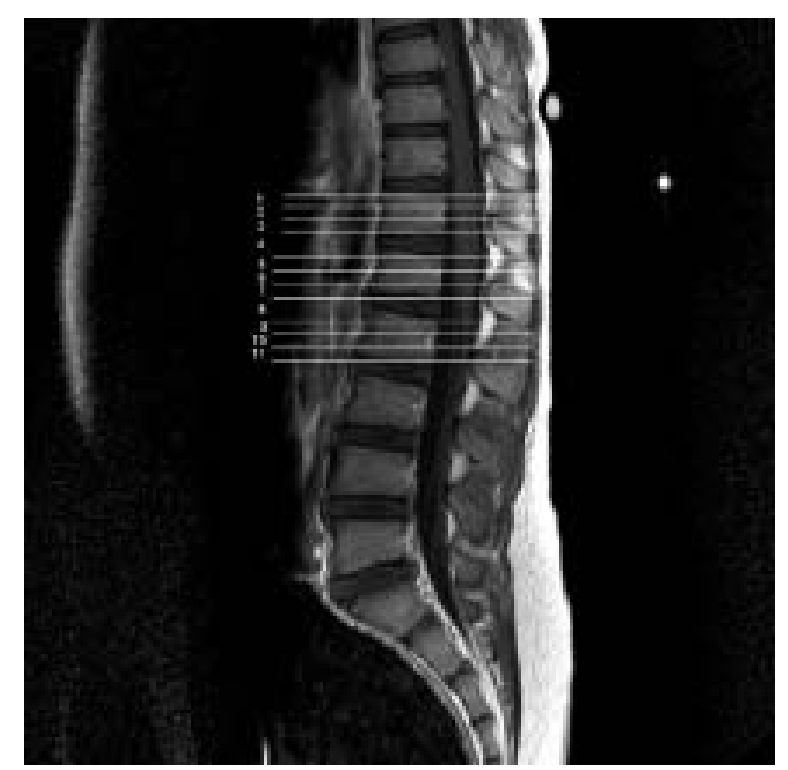

FIG. 3. Sagittal T1-weighted MR image showing the numerical levels (1-11) used for calculating mean, median, and CIs for CM termination. 


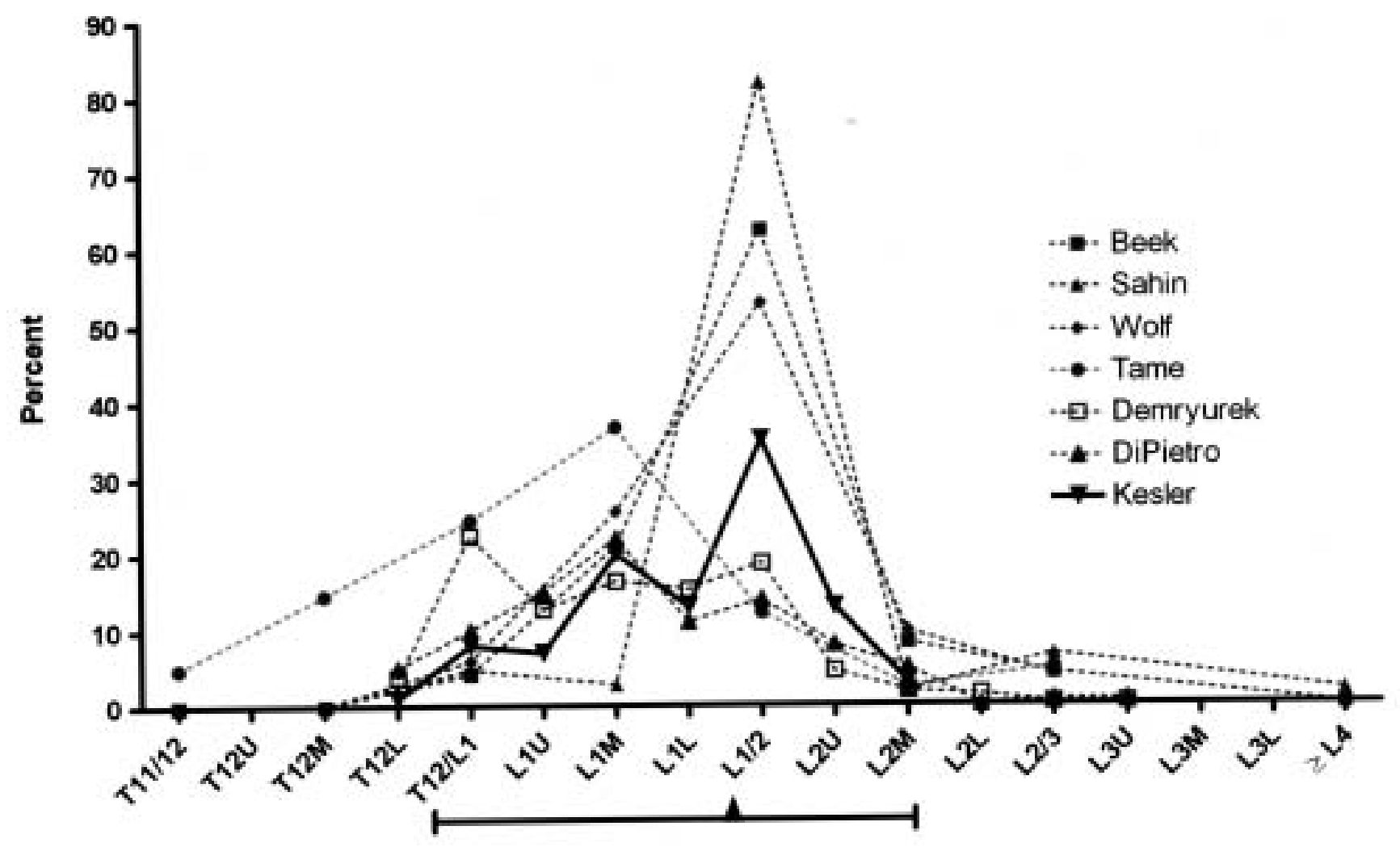

\section{Vertebral Level}

FIG. 4. Distribution of CM termination levels in published studies. Note the presence of the "tail" on the right side of the graph (representing findings caudal to the L1/2 disc space) in previous studies and the absence of such findings in the present study. The overall median value and $95 \%$ CI are indicated by the pointer and line located below the abscissa of the graph.

bearing segments; one of these children had five and the other, six lumbar vertebrae. In both of these children, the $\mathrm{CM}$ ended opposite the middle of the first non-rib-bearing segment. Counting down from the atlas, this vertebra would have been the 12th thoracic vertebra (if it had a rib) in both children, although counting up from the bottom would yield a CM termination level of L1M for the child with five lumbar vertebrae and T12M for the child with six lumbar vertebrae.

Fat was found in the terminal fila of two children, in neither of whom was the filum enlarged. The CM ended at the L1/2 disc space in the first child and at L1M in the second.

\section{Discussion}

This is the first study in which the termination of the $\mathrm{CM}$ has been systematically evaluated in children with reference to the entire spine. Moreover, in this study the evaluation was in children who were not selected for MR imaging evaluations based upon symptoms that might be related to tethering, and the effect of transitional vertebrae on the level of termination of the CM was also evaluated. The results show a much tighter grouping of the level of CM termination between T12L and L2M than has been shown in other studies, with a mode at the L1/2 disc space and a mean being slightly higher at L1L. Of greater importance is that no patient had a CM ending lower than the middle third of the L2 vertebra (L2M). This finding contrasts with those of earlier studies, which have all shown a small subset of patients with CM extending more caudally, ${ }^{3,4,6,9-13}$ even as low as L4 in one series, ${ }^{14}$ despite showing mean levels similar to ours. The inclusion of patients being evaluated for back pain and sciatica in these studies suggests that some of the individuals classified as normal may, in fact, have had symptomatic spinal cord tethering. We conclude that the CM should normally end opposite or cranial to the mid-body of L2 and that any CM that lies caudal to this level should be considered tethered in terms of imaging findings.

The incidence of transitional vertebrae in this study is comparable to the $6 \%$ reported in other series. The results suggest that the presence of a transitional vertebra does not alter the level of termination of the CM if there are 12 rib-bearing segments and the first non rib-bearing segment is identified as L1. Moreover, even in children with 11 ribs, the $\mathrm{CM}$ appears to end in the region of the first non-rib-bearing segment (which would be T12 counting down from the atlas) rather than at $\mathrm{L} 1$. The examination of patients with both transitional vertebrae and reduced 
numbers of rib-bearing segments suggests that the termination of the CM may be regulated by the level of the last rib-bearing segment, a hypothesis that requires further study.

Finally, the results of this study suggest that the use of gelatin capsules to transition from cervicothoracic to thoracolumbar MR images may not always be accurate, as the capsules appear to have moved slightly between the studies in two of the children in this series. This movement caused a misreading on the initial MR imaging interpretation in both patients. We suggest that anatomical landmarks are more suited for aligning the vertebrae between studies.

\section{References}

1. Barson AJ: The vertebral level of termination of the spinal cord during normal and abnormal development. J Anat 106: 489-497, 1970

2. Beek FJ, de Vries LS, Gerards LJ, Mali WP: Sonographic determination of the position of the conus medullaris in premature and term infants. Neuroradiology 38 (1 Suppl): S174-S177, 1996

3. Demiryürek D, Aydingöz U, Aksit MD, Yener N, Geyik PO: MR imaging determination of the normal level of conus medullaris. J Clin Imaging 26:375-377, 2002

4. DiPietro MA: The conus medullaris: normal US findings throughout childhood. Radiology 188:149-153, 1993

5. Hawass ND, el-Badawi MG, Fatani JA, Meshari AA, Abbas FS, Edrees YB, et al: Myelographic study of the spinal cord ascent during fetal development. AJNR Am J Neuroradiol 8: 691-695, 1987

6. Hill CA, Gibson PJ: Ultrasound determination of the normal location of the conus medullaris in neonates. AJNR Am J Neuroradiol 16:469-472, 1994

7. James CCM, Lassman LP: Spinal Dysraphism: Spina Bifida Occulta. London: Butterworth, 1972

8. Jit I, Charnalia VM: The vertebral level of termination of the spinal cord. J Anat Soc India 8:93-102, 1959

9. Lee CH, Seo BK, Choi YC, Shin HJ, Park JH, Jeon HJ, et al: Using MRI to evaluate anatomic significance of aortic bifurcation, right renal artery, and conus medullaris when locating lumbar vertebral segments. AJR Am J Roradiol 182:12951300, 2004

10. Sahin F, Selçuki M, Ecin N, Zenciroglu A, Unlü A, Yilmaz F, et al: Level of conus medullaris in term and preterm neonates. Arch Dis Child Fetal Neonatal Ed 77: F67-F69, 1997

11. Saifuddin A, Burnett SJ, White J: The variation of position of the conus medullaris in an adult population. A magnetic resonance imaging study. Spine 23:1452-1456, 1998

12. Tame SJ, Burstal R: Investigation of the radiological relationship between iliac crests, conus medullaris and vertebral level in children. Paediatr Anaesth 13:676-680, 2003

13. Wilson DA, Prince JR: John Caffey award. MR imaging determination of the location of the normal conus medullaris throughout childhood. AJR Am J Radiol 152:1029-1032, 1989

14. Wolfe S, Schneble F, Tröger J: The conus medullaris: time of ascendence to normal level. Pediatr Radiol 22:590-592, 1992

Manuscript submitted May 6, 2007.

Accepted June 22, 2007.

Current address for Dr. Kesler: Strong Memorial Hospital, Rochester, New York.

Address reprint requests to: Mark S. Dias, M.D., Department of Neurosurgery H 110, Penn State Milton S. Hershey Medical Center, 500 University Drive, Hershey, Pennsylvania 17033. email: mdias @psu.edu. 
Author Names, et al. 